\begin{tabular}{|c|c|}
\hline Title & $\begin{array}{l}\text { Precise thickness control in recess etching of A IGaN/GaN hetero-structure using photocarrier-regulated electrochemical } \\
\text { process }\end{array}$ \\
\hline Author(s) & Kumazaki, Y usuke; Uemura, Keisuke; Sato, Taketomo; Hashizume, Tamotsu \\
\hline Citation & $\begin{array}{l}\text { Journal of A pplied Phy sics, 121(18), } 184501 \\
\text { https://doi.org/10.1063/1.4983013 }\end{array}$ \\
\hline Issue Date & 2017-05-14 \\
\hline Doc URL & http:/hdl.handle.net/2115/70195 \\
\hline Rights & $\begin{array}{l}\text { The following article appeared in Journal of A pplied Physics } 121,184501 \text { (2017) and may be found at } \\
\text { http://aip.scitation.org/doi } / 10.1063 / 1.4983013 \text {. }\end{array}$ \\
\hline Type & article \\
\hline File Information & 1\% 2E4983013.pdf \\
\hline
\end{tabular}

Instructions for use 


\section{Precise thickness control in recess etching of AIGaN/GaN hetero-structure using photocarrier-regulated electrochemical process}

Yusuke Kumazaki, Keisuke Uemura, Taketomo Sato, and Tamotsu Hashizume

Citation: Journal of Applied Physics 121, 184501 (2017); doi: 10.1063/1.4983013

View online: http://dx.doi.org/10.1063/1.4983013

View Table of Contents: http://aip.scitation.org/toc/jap/121/18

Published by the American Institute of Physics

\section{Articles you may be interested in}

Direct observation of inclined a-type threading dislocation with a-type screw dislocation in GaN Journal of Applied Physics 121, 185101 (2017); 10.1063/1.4983254

Trench formation and corner rounding in vertical GaN power devices Applied Physics Letters 110, 193506 (2017); 10.1063/1.4983558

Metal-organic chemical vapor deposition of high quality, high indium composition N-polar InGaN layers for tunnel devices

Journal of Applied Physics 121, 185707 (2017); 10.1063/1.4983300

Improved on-state performance of AIGaN/GaN Fin-HEMTs by reducing the length of the nanochannel

Applied Physics Letters 110, 193502 (2017); 10.1063/1.4983557

Molecular dynamics studies of defect formation during heteroepitaxial growth of InGaN alloys on (0001) GaN surfaces

Journal of Applied Physics 121, 195301 (2017); 10.1063/1.4983066

High temperature operation of $\mathrm{n}$-AIGaN channel metal semiconductor field effect transistors on low-defect AIN templates

Applied Physics Letters 110, 193501 (2017); 10.1063/1.4982656

\section{A|P| $\begin{aligned} & \text { Journal of } \\ & \text { Applied Physics }\end{aligned}$}

Save your money for your research.

It's now FREE to publish with us no page, color or publication charges apply.
Publish your research in the

Joumal of Applied Physics

to claim your place in applied

physics history. 


\title{
Precise thickness control in recess etching of AIGaN/GaN hetero-structure using photocarrier-regulated electrochemical process
}

\author{
Yusuke Kumazaki, Keisuke Uemura, Taketomo Sato, and Tamotsu Hashizume \\ Research Center for Integrated Quantum Electronics, and Graduate School of Information Science \\ and Technology, Hokkaido University, Sapporo 060-8628, Japan
}

(Received 10 March 2017; accepted 22 April 2017; published online 8 May 2017)

\begin{abstract}
The photocarrier-regulated electrochemical (PREC) process was developed for fabricating recessed-gate AlGaN/GaN high-electron-mobility transistors (HEMTs) for normally off operation. The PREC process is based on photo-assisted electrochemical etching using low-energy chemical reactions. The fundamental photo-electrochemical measurements on AlGaN/GaN heterostructures revealed that the photo-carriers generated in the top AlGaN layer caused homogeneous etching of $\mathrm{AlGaN}$ with a smooth surface, but those generated in the GaN layer underneath caused inhomogeneous etching that roughens the surface. The concept of the PREC process is to supply the photocarriers generated only in the AlGaN layer by selecting proper conditions on light wavelength and voltage. The phenomenon of self-termination etching has been observed during the PREC process, where the etching depth was controlled by light intensity. The recessed-gate AlGaN/GaN HEMT fabricated with the PREC process showed positive threshold voltage and improvement in transconductance compared to planar-gate AlGaN/GaN HEMTs. Published by AIP Publishing.

[http://dx.doi.org/10.1063/1.4983013]
\end{abstract}

\section{INTRODUCTION}

AlGaN/GaN high-electron-mobility transistors (HEMTs) are promising candidates for high-power electronics applications because high blocking voltage yet low on-resistance $\left(R_{\mathrm{ON}}\right)$ can be achieved. ${ }^{1-4}$ From the fail-safe viewpoint of power-switching devices, it is necessary to attain normally off operation. One of the promising approaches to attain such operation is adopting a recessed-gate structure, ${ }^{5-7}$ which can be fabricated by thinning the AlGaN layer beneath the gate electrode. The dry etching process is commonly used for thinning the AlGaN layer because the wet etching process is not applicable due to the chemical stability of group-III nitrides. ${ }^{8-10}$ However, dry-etched surfaces are generally negatively affected by various types of damages, which may lead to degradation of the device's performance. ${ }^{11,12}$ Moreover, unintentional variations in the recess depth make it difficult to precisely control the threshold voltage $\left(V_{\text {th }}\right)$.

One alternative approach is photo-assisted electrochemical (PEC) etching, which is a cyclic process consisting of anodic oxidation and subsequent dissolution of the resulting oxide in an electrolyte. Compared to dry etching, PEC etching is highly desirable in its simplicity and the absence of plasma damage. ${ }^{13,14}$ Also, this etching is applicable to various semiconductors, even chemically stable materials such as group-III nitrides. ${ }^{15}$

Recessed-gate AlGaN/GaN HEMTs using PEC etching have recently been reported. Chiou et al. ${ }^{16}$ improved transconductance $\left(g_{\mathrm{m}}\right)$ by PEC etching and subsequent PEC oxidation. Zhang et al. ${ }^{17}$ showed the suitability of PEC etching in ionic liquid etchant by developing high-performance normally off HEMTs. We previously attained normally off operation in recessed-oxide gate structures formed by PEC oxidation in parallel with PEC etching conducted in the same electrolyte. ${ }^{18}$
Despite the fact that device performances of recessed-gate AlGaN/GaN HEMTs fabricated by PEC etching have been demonstrated, important aspects for recess etching, such as depth-controllability and surface roughness, have not been sufficiently investigated. Although PEC etching is caused by photo-carriers, carrier transfer in an AlGaN/GaN heterostructure is not fully understood, which makes it difficult to optimize electrochemical conditions to obtain desirable etching features.

In this study, we investigated the basic photoelectrochemical behavior of an AlGaN/GaN hetero-structure under monochromatic light to clarify the carrier-transfer process in PEC etching. Based on the photocarrier-regulated electrochemical (PREC) process, we succeeded in selfterminating and depth-controllable etching of an AlGaN/ $\mathrm{GaN}$ hetero-structure with a very smooth surface. We also examined the electrical properties of a recessed-gate AlGaN/ GaN HEMT fabricated using the PREC process.

\section{METHODS}

An $\mathrm{i}-\mathrm{Al}_{0.25} \mathrm{Ga}_{0.75} \mathrm{~N} / \mathrm{i}-\mathrm{GaN}$ hetero-structure grown on a $\mathrm{Si}$ substrate was used as the starting wafer, as shown in Fig. 1(a). The thickness of the $\mathrm{AlGaN}$ barrier layer was $25 \mathrm{~nm}$. A Ti/Al/Ti/Au (20/50/20/50 nm) multilayer ohmic electrode was formed using the electron-beam evaporation method, followed by annealing at $830{ }^{\circ} \mathrm{C}$ for $1 \mathrm{~min}$ in a $\mathrm{N}_{2}$ ambient atmosphere. Then, $\mathrm{a} \mathrm{SiO}_{2}$ film $(100 \mathrm{~nm})$ was formed by sputtering and lithography to define the etching region. We carried out PEC etching using a three-electrode cell (Fig. 1(b)), which consisted of the sample to be etched as the working electrode (WE), Pt counter electrode (CE), and $\mathrm{Ag} / \mathrm{AgCl}$ reference electrode $(\mathrm{RE})$, immersed in a mixture of $1 \mathrm{~mol} / \mathrm{L}$ $\mathrm{H}_{2} \mathrm{SO}_{4}$ and $1 \mathrm{~mol} / \mathrm{L} \mathrm{H}_{3} \mathrm{PO}_{4}(\mathrm{pH}=1.7)$ as the electrolyte. The 
(a)

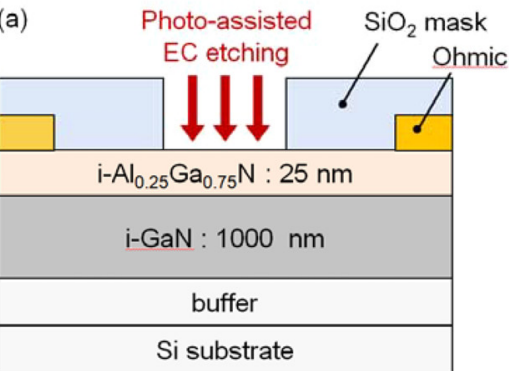

(b)

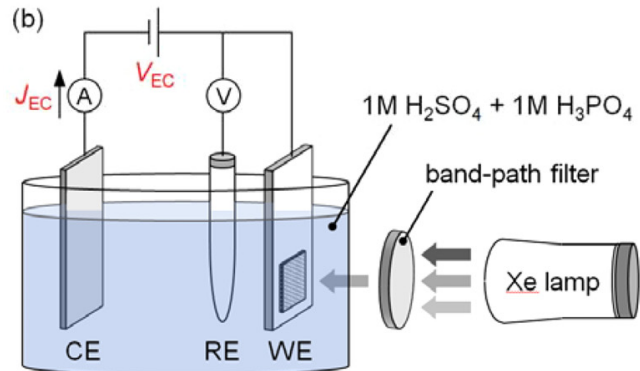

FIG. 1. Schematic representations of the (a) sample structure, and (b) experimental setup of photo-assisted electrochemical (PEC) etching. ohmic contact was electrically connected to an outer circuit, in which the potential of WE was controlled with respect to the RE by using a potentiostat with a Princeton Applied Research VersaSTAT 4. Xenon (Xe) lamp as the light source, and monochromatic light passing through band-path filters was irradiated from the top of the WE.

Prior to etching experiments, the basic photoelectrochemical behavior of the $\mathrm{AlGaN} / \mathrm{GaN}$ hetero-structure was investigated by measuring the current under monochromatic light. Considering the photo-electrochemical behavior with potential distribution, the relationships between electrochemical reactions and carrier-transfer processes were also investigated. Based on the regulation of carrier transfer, two types of PEC etching conditions were compared to investigate the effect of the carrier-transfer process on etching behavior. We then fabricated as Schottky diode and Schottky-gate HEMTs to evaluate the electrical properties of the AlGaN/ $\mathrm{GaN}$ hetero-structure samples after etching.

\section{RESULTS AND DISCUSSION}

\section{A. Photo-electrochemical properties of the AIGaN/GaN hetero-structure}

Figure 2 shows the current-voltage $\left(J_{\mathrm{EC}}-V_{\mathrm{EC}}\right)$ characteristics of the AlGaN/GaN hetero-structure immersed in the

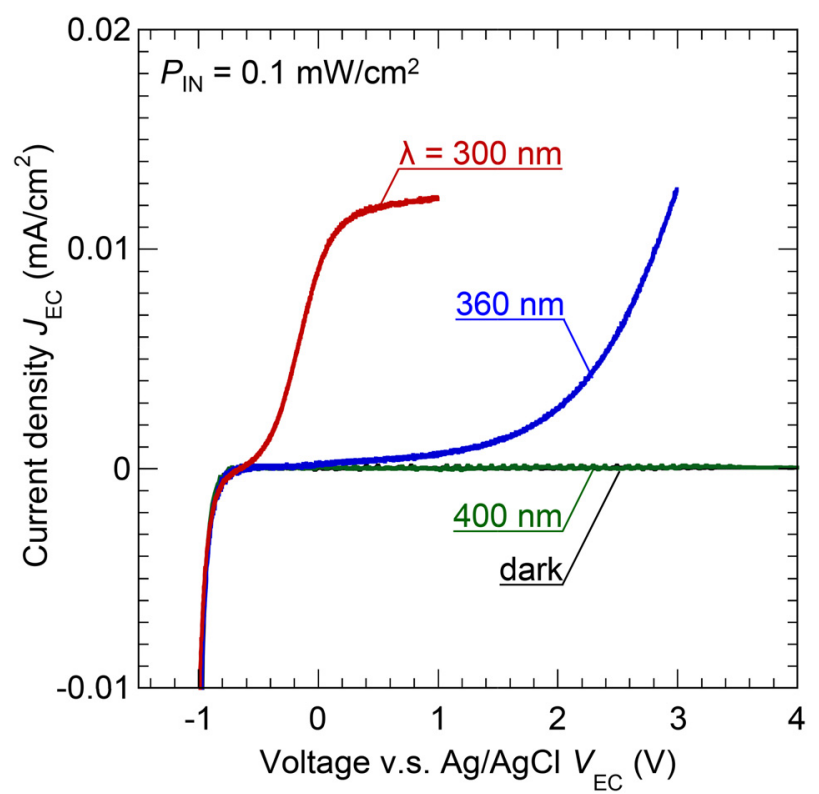

FIG. 2. Current-voltage characteristics of the AlGaN/GaN hetero-structure immersed in the electrolyte in the dark (a black line) and under light of wavelength $(\lambda)=300$ (red line), 360 (blue line), and $400 \mathrm{~nm}$ (green line). The sweep direction was positive, and the sweep rate was set to $50 \mathrm{mV} / \mathrm{s}$. electrolyte in the dark (a black line) and under light of wavelengths $(\lambda)$ of 300 (red line), 360 (blue line), and $400 \mathrm{~nm}$ (green line). Figure 3 shows the potential distribution of the electrolyte/AlGaN/GaN structure at voltages $\left(V_{\mathrm{EC}}\right)$ of (a) -1.0 , (b) 1.0 , and (c) $3.0 \mathrm{~V}$, calculated using the onedimensional Poisson equation.

Negative currents (reduction currents) were observed at potentials negative to around $-0.8 \mathrm{~V}$ under all light conditions. Since the surface potential of $\mathrm{AlGaN}$ becomes low by applying negative voltage, as shown in Fig. 3(a), the electrons in two-dimensional electron gas (2DEG) flow toward the electrolyte/AlGaN interface and cause reduction reactions. The electron concentration in 2DEG is generally as high as $10^{19} \mathrm{~cm}^{-3}$, a reason why the reduction currents were barely affected by light illumination. At the positive voltage, few currents were observed on the $\mathrm{AlGaN} / \mathrm{GaN}$ hetero-structure in

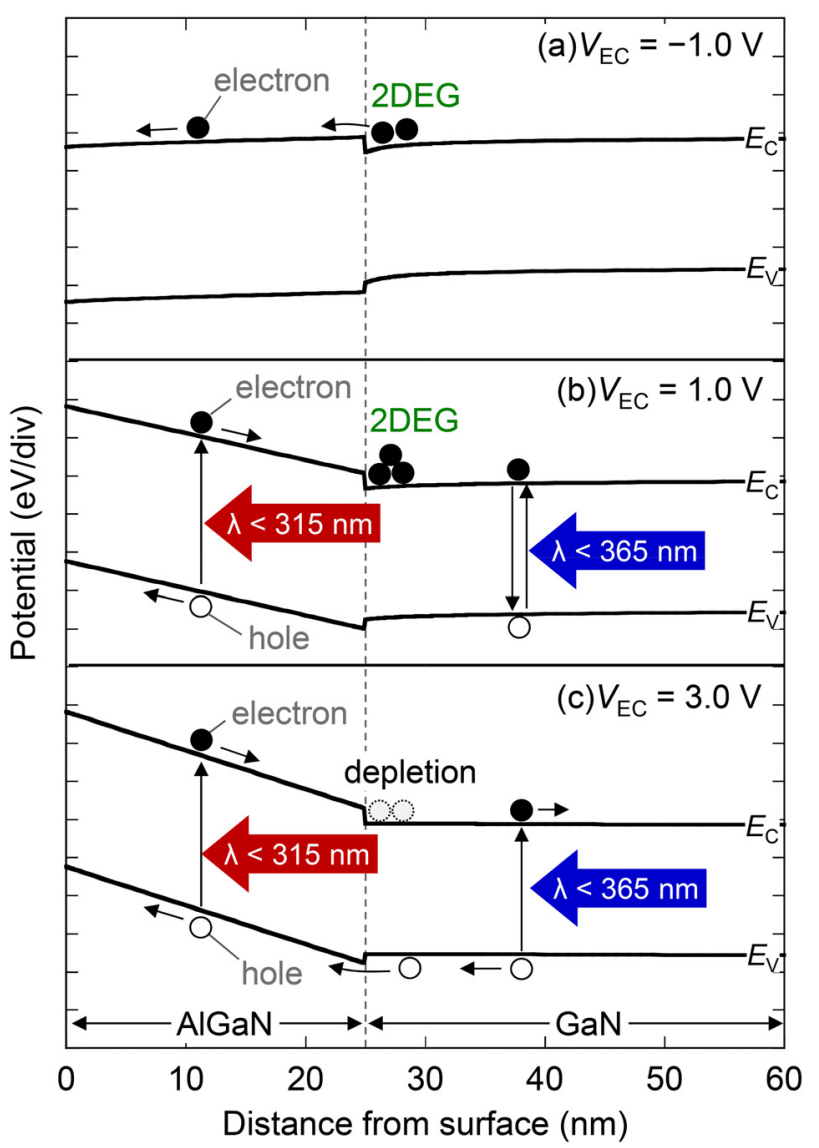

FIG. 3. Potential distribution of the electrolyte/AlGaN/GaN structure at voltage $\left(V_{\mathrm{EC}}\right)$ of (a) -1.0 , (b) 1.0 , and (c) 3.0 calculated using the onedimensional Poisson equation. The Schottky barrier height was assumed to be $1.0 \mathrm{eV}$. 
the dark and under light of $\lambda=400 \mathrm{~nm}$ since electron flow was restricted due to the AlGaN barrier layer, and few holes existed in both $\mathrm{AlGaN}$ and GaN layers. Although light of $\lambda=400 \mathrm{~nm}$ was absorbed in the $\mathrm{Si}$ substrate, photo-carriers could not flow due to the existence of the high-resistive buffer layer. This rectifying behavior observed on the $\mathrm{AlGaN} / \mathrm{GaN}$ hetero-structure was similar to the n-type semiconductor electrodes in the dark, where positive current behaviors by photocarriers are quite different. Under light of $\lambda=360 \mathrm{~nm}$, which penetrates the $\mathrm{AlGaN}$ layer and is absorbed in the $\mathrm{GaN}$ layer, positive current (oxidation current) was observed with $V_{\mathrm{EC}}$ larger than around $1.0 \mathrm{~V}$. Considering the potential distribution at $V_{\mathrm{EC}}=1.0 \mathrm{~V}$ (Fig. 3(b)), electrons existed in 2DEG with high concentration, which prevented photo-holes from flowing toward the electrolyte/AlGaN interface; photo-carriers resulted in recombination and little current for surface oxidation was observed at $V_{\mathrm{EC}}=1.0 \mathrm{~V}$. The amount of electrons concentrated in 2DEG, however, decreased with an increase in $V_{\mathrm{EC}}$ and fully depleted at $V_{\mathrm{EC}}=3.0 \mathrm{~V}$, as shown in Fig. 3(c). In such a situation, photo-holes can flow toward the electrolyte/AlGaN interface and cause oxidation reactions; thus, oxidation current increases rapidly at around $V_{\mathrm{EC}}=3.0 \mathrm{~V}$, as shown in Fig. 2. Obviously, oxidation reactions under this condition are caused by photo-holes generated in the $\mathrm{GaN}$ layer.

Under light of $\lambda=300 \mathrm{~nm}$, which is absorbed in both $\mathrm{AlGaN}$ and GaN layers, oxidation current started to flow at around $V_{\mathrm{EC}}=-0.5 \mathrm{~V}$, and subsequently saturated at around $V_{\mathrm{EC}}=0 \mathrm{~V}$. Since strong electric field exists in the AlGaN layer even at low $V_{\mathrm{EC}}$, as shown in Fig. 3(b), photo-electrons and holes were transferred to the 2DEG and electrolyte/ AlGaN interface, respectively; thus, oxidation current flowed even at relatively low $V_{\mathrm{EC}}$. As previously discussed, photocarriers generated in the $\mathrm{GaN}$ layer cannot flow at $V_{\mathrm{EC}}$ below $1.0 \mathrm{~V}$ due to the existence of a high concentration of electrons in the 2DEG; thus, it is obvious that oxidation reactions under this condition are caused by photo-holes generated in the AlGaN layer.

Thus, we found that oxidation reactions on the AlGaN/ $\mathrm{GaN}$ hetero-structure are caused by either photo-holes generated in the GaN layer or those generated in the AlGaN layer, or both. In addition, we can regulate the supply of photoholes by selecting the appropriate $\lambda$ and $V_{\mathrm{EC}}$. In Section III B, we discuss the importance of photo-hole regulation in achieving homogeneous PEC etching.

\section{B. PEC etching based on regulation of photo-carriers}

We first carried out PEC etching by using photo-holes generated in the GaN layer. Figure 4(a) shows an atomicforce microscopy (AFM) image of a sample after PEC etching with $V_{\mathrm{EC}}=5.0 \mathrm{~V}, \lambda=360 \mathrm{~nm}$, light intensity $\left(P_{\mathrm{IN}}\right)=1.0 \mathrm{~mW} /$ $\mathrm{cm}^{2}$, and etching time $\left(t_{\mathrm{EC}}\right)=16 \mathrm{~min}$. The $\mathrm{SiO}_{2}$ film used for defining the etching region was completely removed by HFbased solution. From the AFM measurement, there was no difference in height between the masked and un-masked regions for etching despite large amounts of oxidation current. From the top scanning electron microscopy (SEM) image shown in Fig. 4(b), however, small pores with diameters of $20 \mathrm{~nm}$ or less could be observed only at an un-masked region,
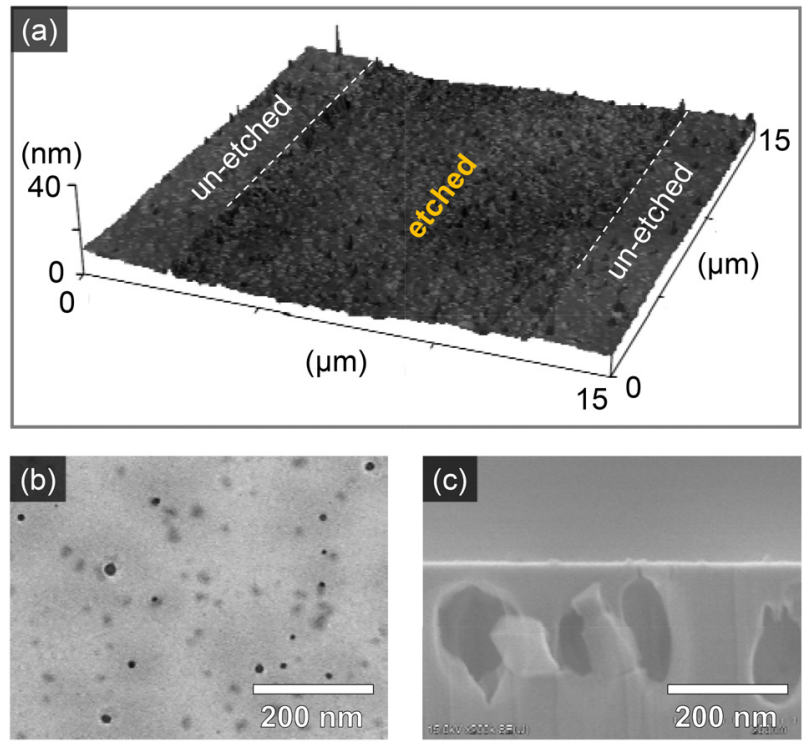

FIG. 4. (a) Three-dimensional atomic-force microscopy (3D-AFM) image, (b) top scanning electron microscopy (SEM) image, and (c) cross-sectional SEM image of the sample (an un-masked region) after PEC etching with photo-carriers generated in the GaN layer: $V_{\mathrm{EC}}=5.0 \mathrm{~V}, \lambda=360 \mathrm{~nm}$, and light intensity $\left(P_{\mathrm{IN}}\right)=1.0 \mathrm{~mW} / \mathrm{cm}^{2}$.

indicating that inhomogeneous etching occurred. Since the pore depth was estimated to be $240 \mathrm{~nm}$ from the crosssectional SEM image shown in Fig. 4(c), pores seemed to pierce the AlGaN layer. The pore diameter in the AlGaN layer was estimated to be $20 \mathrm{~nm}$ or less as was the case with that estimated from the top SEM image, whereas the pore diameter in the GaN layer was estimated to be about 50 to $100 \mathrm{~nm}$. An increase in $t_{\mathrm{EC}}$ leads to an increase in the pore diameter in the GaN layer (pore diameter in the $\mathrm{AlGaN}$ layer remained unchanged), indicating that carrier transfer at the electrolyte/GaN interface occurred rather than at the electrolyte/AlGaN interface.

From the above results, we can conclude that photoholes generated in the GaN layer cause inhomogeneous etching. Generally, inhomogeneous etching of a semiconductor results from localized carrier transfer by high-electric field applied at the electrolyte/semiconductor interface. ${ }^{19-21}$ Similar to the case with the carrier transfer in a Schottky barrier diode ${ }^{22-25}$ localized carrier transfer may be caused by inhomogeneous potential distribution due to the concentration of the electric field on crystallographic disorders such as dislocations and vacancies. Since inhomogeneous etching observed under this condition will be the origin of rough surfaces, the use of photo-holes generated in the GaN layer is unfavorable for the fabrication of recessed-gate structures.

Next, we carried out PEC etching by using photo-holes generated in the AlGaN layer. Figure 5(a) shows an AFM image of a sample after PEC etching with $V_{\mathrm{EC}}=-0.2 \mathrm{~V}$, $\lambda=300 \mathrm{~nm}, P_{\mathrm{IN}}=1.0 \mathrm{~mW} / \mathrm{cm}^{2}$, and $t_{\mathrm{EC}}=80 \mathrm{~min}$. The etched step could be clearly observed between the masked and un-masked regions, unlike the sample etched by photoholes generated in the GaN layer. The root mean square of the etched region was only $0.41 \mathrm{~nm}$. Figures 5(b) and 5(c) show the top and cross-sectional SEM images of the sample etched under this condition. There seem to be no pores and 

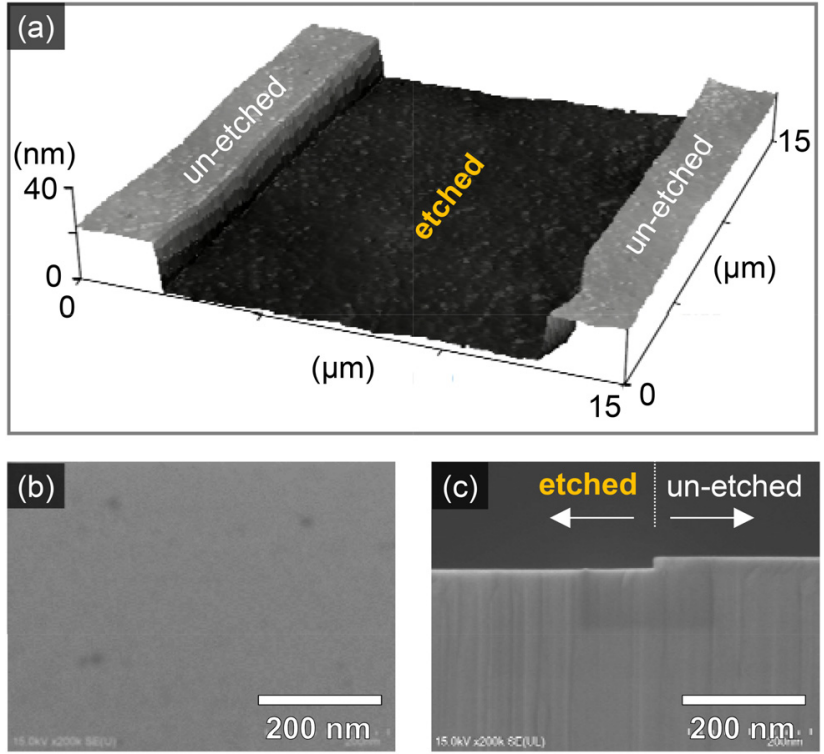

FIG. 5. (a) 3D-AFM image, (b) top SEM image, and (c) cross-sectional SEM image of the sample after PEC etching with photo-carriers generated in the AlGaN layer: $V_{\mathrm{EC}}=-0.2 \mathrm{~V}, \lambda=300 \mathrm{~nm}$, and $P_{\mathrm{IN}}=1.0 \mathrm{~mW} / \mathrm{cm}^{2}$.

no irregularity on the etched region, indicating that homogeneous etching can be achieved by using photo-holes generated in the AlGaN layer.

Figure 6(a) shows the relationship between the etching depth and $t_{\mathrm{EC}}$ obtained on the sample etched with $P_{\mathrm{IN}}=0.5$ $\mathrm{mW} / \mathrm{cm}^{2}$. Etching depth increased linearly with $t_{\mathrm{EC}}$ at the initial stage. However, we found that etching was terminated at a specific depth below the initial AlGaN-layer thickness of $25 \mathrm{~nm}$. In other words, self-termination of etching was observed in the process of AlGaN-layer etching. Figure 6(b) shows the relationship between the self-termination depth and $P_{\mathrm{IN}}$. We found that the self-termination depth increased linearly with $P_{\mathrm{IN}}$, indicating that this phenomenon is related to the amount of photo-holes generated in the AlGaN layer.

From the above results, we considered a mechanism of PEC etching that involves photo-holes generated in the AlGaN layer. As previously mentioned, photo-holes generated in the AlGaN layer are transferred to the electrolyte/AlGaN interface by internal polarization. They cause oxidation reactions of $\mathrm{AlGaN}$, and the resulting oxides, such as $\mathrm{Ga}_{2} \mathrm{O}_{3}$ and $\mathrm{Al}_{2} \mathrm{O}_{3}$, dissolve in the electrolyte, leading to etching of the AlGaN layer. Since photo-holes generated in the AlGaN layer decrease due to the thinning of the layer, etching reactions are suppressed at a specific AlGaN-layer thickness, which cannot generate sufficient photo-holes for surface oxidation; thus, a self-termination phenomenon occurred. Since the amount of photo-holes varies with $P_{\mathrm{IN}}$, the self-terminating depth is a function of $P_{\mathrm{IN}}$, as shown in Fig. 6(b).

Thus, the regulation of photo-holes critically affects etching behavior. Homogeneous etching can be achieved by preventing photo-holes generated in the GaN layer from participating in oxidation reactions. In addition, PEC etching involving photo-holes generated in the AlGaN layer is terminated spontaneously, and the self-termination depth can be controlled by $P_{\mathrm{IN}}$. The self-termination phenomenon may help in suppressing unintentional variations in recess depth.
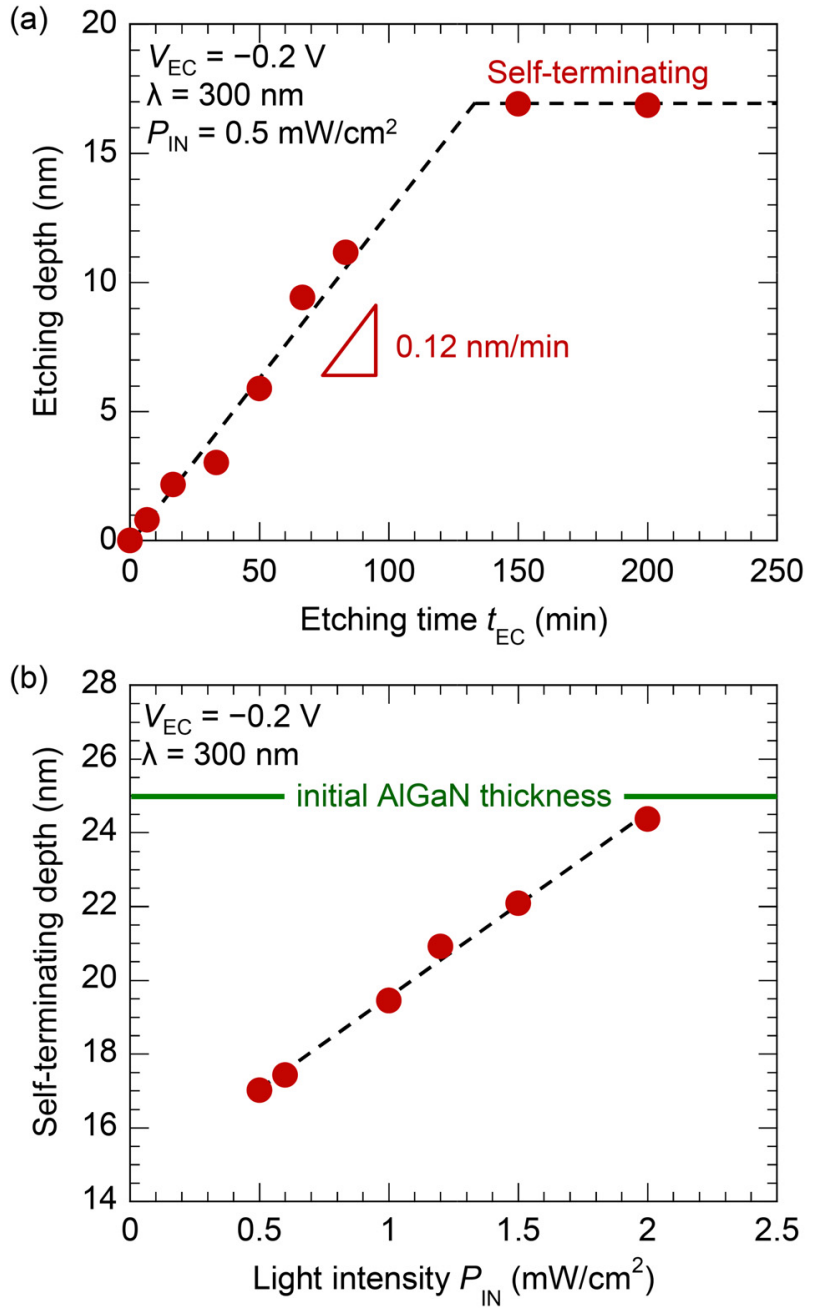

FIG. 6. (a) Relationship between the etching depth and etching time $\left(t_{\mathrm{EC}}\right)$, and (b) relationship between the self-termination depth and $P_{\text {IN }}$ obtained from the sample etched with photo-holes generated in the AlGaN layer.

Thus, PEC etching based on the regulation of photo-holes, i.e., the PREC process, has attractive features for use in fabricating recessed-gate structures.

\section{Electrical properties of recessed-gate AIGaN/GaN HEMTs formed using the PREC process}

We evaluated the electrical properties of PREC-etched AlGaN/GaN hetero-structures by fabricating a Schottky diode and Schottky-gate HEMT. The AlGaN-layer thickness of the un-etched and PREC-etched regions is 25 and $8 \mathrm{~nm}$, respectively.

Figure 7 shows the capacitance-voltage characteristics measured at $100 \mathrm{kHz}$ for Schottky diodes fabricated on planar (black) and PREC-etched (red) samples: symbols represent experimental results, and solid lines represent theoretical curves, assuming an AlGaN-layer thickness of $25 \mathrm{~nm}$ for the planar sample and $8 \mathrm{~nm}$ for the PREC-etched sample. The $V_{\text {th }}$ and saturated capacitance value increased by thinning the AlGaN layer as expected. The experimental data were well reproduced by the theoretical curve, and no hysteresis was observed between the positive and negative sweep directions, 


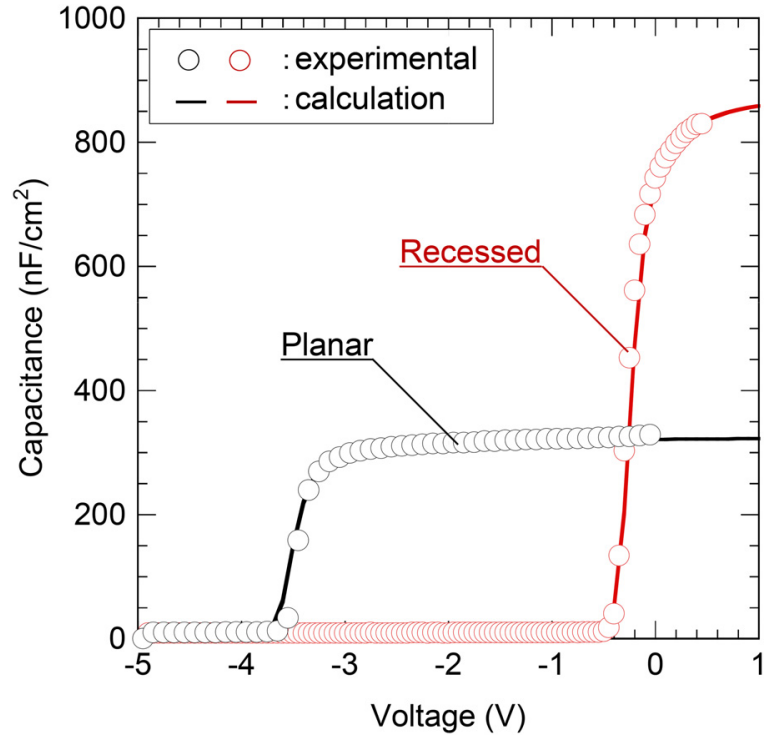

FIG. 7. Capacitance-voltage characteristics measured at $100 \mathrm{kHz}$ for the Schottky diode fabricated on planar (black) and PREC-etched (red) samples: symbols represent experimental results, and solid lines represent the theoretical curve assuming an AlGaN-layer thickness of $25 \mathrm{~nm}$ for the planar sample and $8 \mathrm{~nm}$ for the PREC-etched sample.

suggesting that a nearly ideal Schottky interface was formed on both samples.

Figures 8(a) and 8(b) show the drain current-voltage $\left(I_{\mathrm{DS}}-V_{\mathrm{DS}}\right)$ characteristics of planar-gate and recessed-gate AlGaN/GaN Schottky HEMTs with a gate length $\left(L_{\mathrm{G}}\right)$ of $10 \mu \mathrm{m}$ and source-drain spacing of $30 \mu \mathrm{m}$. Both samples showed good $I-V$ curves with constant saturation currents and pinch-off behavior. The transfer characteristics of the planar-gate and recessed-gate AlGaN/GaN Schottky HEMT in the saturated region $\left(V_{\mathrm{DS}}=10 \mathrm{~V}\right)$ are compared in Fig. 9. The $V_{\text {th }}$ determined using the linear extrapolation method was -2.42 and $+0.30 \mathrm{~V}$ for the planar-gate and recessedgate HEMTs, respectively, as shown in Fig. 9(a). The positive shift of $V_{\text {th }}$ could be confirmed with the recessed-gate

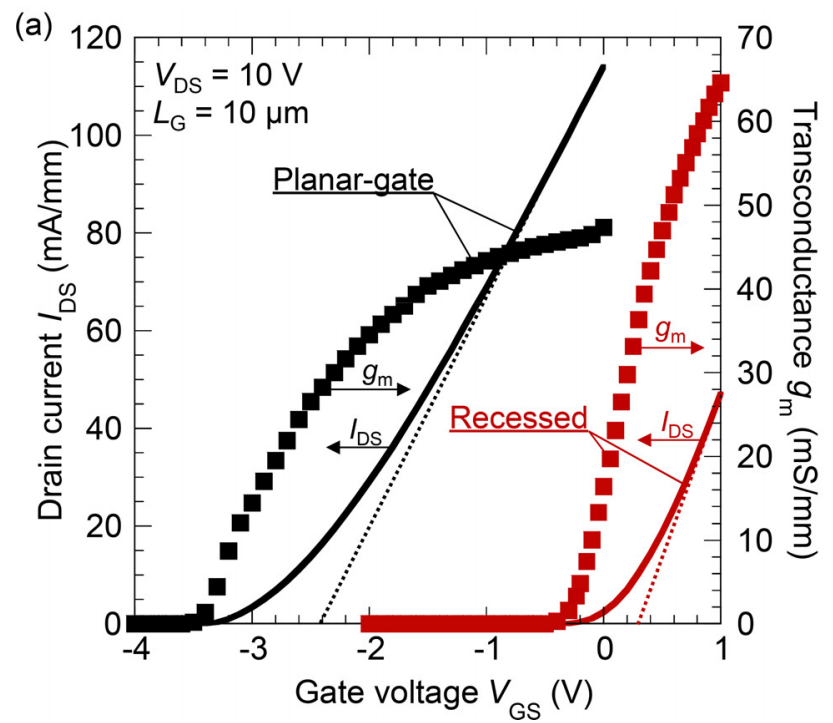

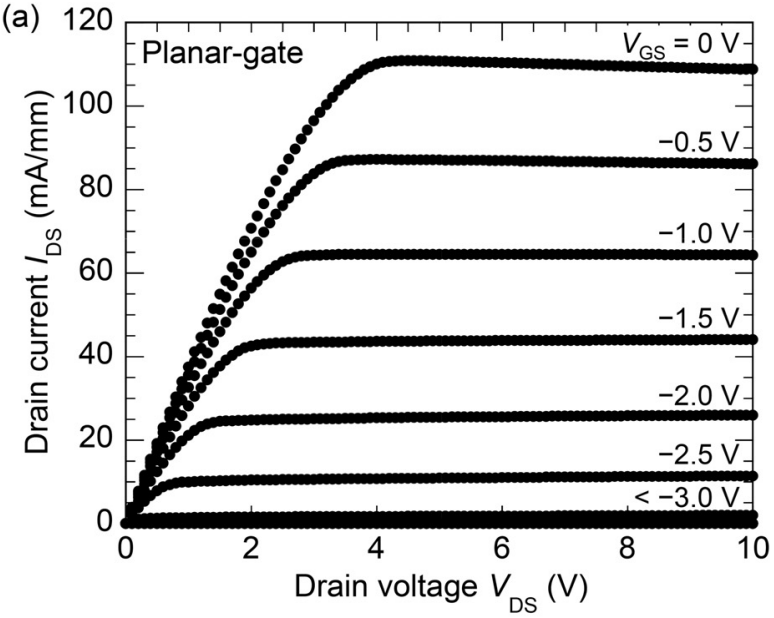

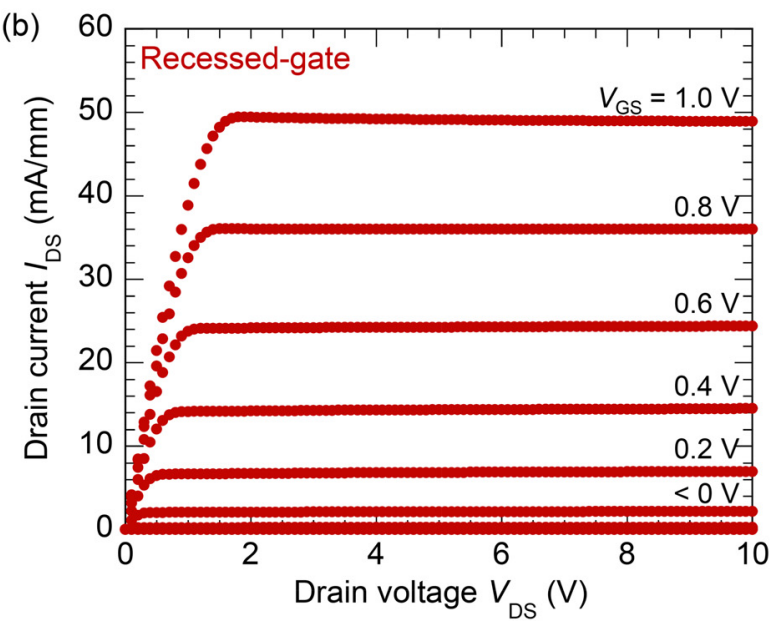

FIG. 8. Drain current-voltage $\left(I_{\mathrm{DS}}-V_{\mathrm{DS}}\right)$ characteristics of (a) planar-gate and (b) recessed-gate AlGaN HEMTs with a gate length $\left(L_{\mathrm{G}}\right)$ of $10 \mu \mathrm{m}$ and source-drain spacing of $30 \mu \mathrm{m}$.

structure, and no hysteresis was observed between positive and negative sweep directions. In addition, the $g_{\mathrm{m}}$ of the recessed-gate HEMT was higher than that of the planar-gate HEMT due to the thinning of the AlGaN layer. In previous

(b)

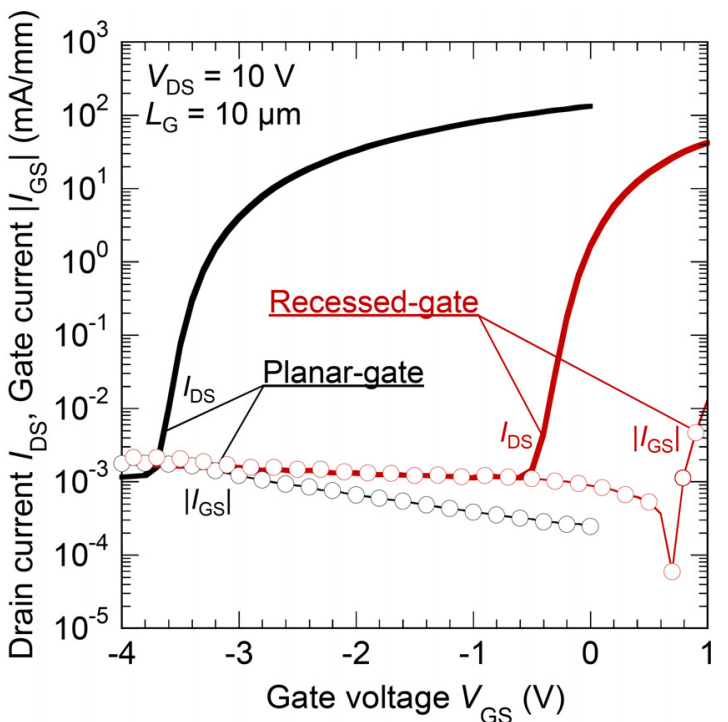

FIG. 9. Transfer characteristics of planar-gate (black) and recessed-gate (red) Schottky HEMTs in the saturated region $\left(V_{\mathrm{DS}}=10 \mathrm{~V}\right)$ : (a) linear plots of $I_{\mathrm{DS}}$ and transconductance $\left(g_{\mathrm{m}}\right)$, and (b) semi-log plots of $I_{\mathrm{DS}}$ and gate current $\left(\left|I_{\mathrm{GS}}\right|\right)$ as a function of gate voltage $\left(V_{\mathrm{GS}}\right)$. 
reports on the recessed-gate HEMTs fabricated by dry etching, the positive $V_{\text {th }}$ was obtained as expected; however, the $g_{\mathrm{m}}$ tended to be degraded due to the increase in gate current $\left(I_{\mathrm{GS}}\right){ }^{26-28}$ On the other hand, both positive $V_{\text {th }}$ and improvement in $g_{\mathrm{m}}$ were simultaneously achieved in the recessedgate HEMT fabricated by the present PREC etching since no significant increase in $I_{\mathrm{GS}}$ was observed after PREC etching as shown in Fig. 9(b). These electrical characteristics suggest that no significant damage was induced in the AlGaN/GaN hetero-structures during PREC etching. We can conclude that PREC etching is desirable for fabricating recessed-gate structures.

\section{CONCLUSIONS}

We investigated the PREC etching process to fabricate recessed-gate AlGaN/GaN HEMTs without inducing damage. The basic photo-electrochemical characteristics of AlGaN/ $\mathrm{GaN}$ hetero-structures revealed that two types of photo-holes generated in either the $\mathrm{AlGaN}$ layer or GaN layer could be supplied to the solid/liquid interface separately by selecting an appropriate light wavelength and voltage. The holeregulation under these conditions significantly affected etching behavior: photo-holes generated in the GaN layer caused inhomogeneous etching and those generated in the $\mathrm{AlGaN}$ layer caused homogeneous etching. In the PREC process, the phenomenon of self-termination etching was also observed. The self-termination depth could be controlled by light intensity, enabling us to obtain the desired AlGaN-layer thickness without unintentional variation. The recessed-gate AlGaN/ GaN HEMT showed positive threshold voltage and improvement in transconductance compared to the planar-gate AlGaN/GaN HEMT. The PREC process, therefore, is very attractive for use in fabricating recessed-gate structures.

\section{ACKNOWLEDGMENTS}

This work was supported in part by a Grant-in-Aid for JSPS Fellows JP14J01371, a Grant-in-Aid for Challenging Exploratory Research JP15K13937, and a Grant-in-Aid for Scientific Research on Innovative Areas JP16H06421, from the Japan Society for the Promotion of Science (JSPS).
${ }^{1}$ Y. Uemoto, M. Hikita, H. Ueno, H. Matsuo, H. Ishida, M. Yanagihara, T. Ueda, T. Tanaka, and D. Ueda, IEEE Trans. Electron Devices 54, 3393 (2007).

${ }^{2}$ T. Kikkawa, K. Makiyama, T. Ohki, M. Kanamura, K. Imanishi, N. Hara, and K. Joshin, Phys. Status Solidi A 206, 1135 (2009).

${ }^{3}$ R. Chu, A. Corrion, M. Chen, R. Li, D. Wong, D. Zehnder, B. Hughes, and K. Boutros, IEEE Electron Device Lett. 32, 632 (2011).

${ }^{4}$ M. Kuzuhara, J. T. Asubar, and H. Tokuda, Jpn. J. Appl. Phys., Part 1 55, 070101 (2016).

${ }^{5}$ T. Palacios, C. S. Suh, A. Chakraborty, S. Keller, S. P. DenBaars, and U. K. Mishra, IEEE Electron Device Lett. 27, 428 (2006).

${ }^{6}$ S. Maroldt, C. Haupt, W. Pletschen, S. Müller, R. Quay, O. Ambacher, C. Schippel, and F. Schwierz, Jpn. J. Appl. Phys., Part 1 48, 04 C083 (2009).

${ }^{7}$ S. Huang, X. Liu, J. Zhang, K. Wei, G. Liu, X. Wang, Y. Zheng, H. Liu, Z. Jin, C. Zhao, C. Liu, S. Liu, S. Yang, J. Zhang, Y. Hao, and K. J. Chen, IEEE Electron Device Lett. 36, 754 (2015).

${ }^{8}$ R. S. Qhalid Fareed, X. Hu, A. Tarakji, J. Deng, R. Gaska, M. Shur, and M. A. Khan, Appl. Phys. Lett. 86, 143512 (2005).

${ }^{9}$ T. Oka and T. Nozawa, IEEE Electron Device Lett. 29, 668 (2008).

${ }^{10}$ N. Maeda, M. Hiroki, S. Sasaki, and Y. Harada, Appl. Phys. Express 5, 084201 (2012).

${ }^{11}$ Z. Mouffak, A. Bensaoula, and L. Trombetta, J. Appl. Phys. 95, 727 (2004).

${ }^{12}$ K. Tang, W. Huang, and T. P. Chow, J. Electron. Mater. 38, 523 (2009).

${ }^{13}$ N. Shiozaki, T. Sato, and T. Hashizume, Jpn. J. Appl. Phys., Part 1 46, 1471 (2007).

${ }^{14}$ N. Shiozaki and T. Hashizume, J. Appl. Phys. 105, 064912 (2009).

${ }^{15}$ T. Sato, Y. Kumazaki, M. Edamoto, M. Akazawa, and T. Hashizume, Proc. SPIE 9748, 97480Y (2016).

${ }^{16}$ Y. L. Chiou, L. H. Huang, and C. T. Lee, IEEE Electron Device Lett. 31, 183 (2010).

${ }^{17}$ Z. Zhang, S. Qin, K. Fu, G. Yu, W. Li, X. Zhang, S. Sun, L. Song, S. Li, and R. Hao, Appl. Phys. Express 9, 084102 (2016).

${ }^{18}$ N. Harada, Y. Hori, N. Azumaishi, K. Ohi, and T. Hashizume, Appl. Phys. Express 4, 021002 (2011).

${ }^{19}$ Y. Kumazaki, A. Watanabe, Z. Yatabe, and T. Sato, J. Electrochem. Soc. 161, H705 (2014).

${ }^{20}$ A. Watanabe, Y. Kumazaki, Z. Yatabe, and T. Sato, ECS Electrochem. Lett. 4, H11 (2015).

${ }^{21}$ Y. Kumazaki, Z. Yatabe, and T. Sato, Jpn. J. Appl. Phys., Part 1 55, 04EJ12 (2016).

${ }^{22}$ O. Mitrofanov and M. Manfra, J. Appl. Phys. 95, 6414 (2004).

${ }^{23}$ H. Zhang, E. J. Miller, and E. T. Yu, J. Appl. Phys. 99, 023703 (2006).

${ }^{24}$ H. Hasegawa and S. Oyama, J. Vac. Sci. Technol. B 20, 1647 (2002).

${ }^{25}$ J. Kotani, T. Tamotsu, and H. Hasegawa, J. Vac. Sci. Technol. B 22, 2179 (2004).

${ }^{26}$ T. J. Anderson, M. J. Tadjer, M. A. Mastro, J. K. Hite, K. D. Hobart, C. R. Eddy, and F. J. Kub, J. Electron. Mater. 39, 478 (2010).

${ }^{27}$ Z. He, J. Li, T. Wen, Z. Shen, Y. Tao, F. Yang, Y. Ni, Z. Wu, B. Zhang, and Y. Liu, Jpn. J. Appl. Phys., Part 1 51, 054103 (2012).

${ }^{28}$ J.-H. Lin, S.-J. Huang, C.-H. Lai, and Y.-K. Su, Jpn. J. Appl. Phys., Part 1 55, $01 \mathrm{AD} 05$ (2016). 